\title{
Corpus callosum agenesis-neuronopathy syndrome
}

INSERM

\section{Source}

INSERM. (1999). Orphanet: an online rare disease and orphan drug data base. Corpus callosum agenesis-neuronopathy syndrome. ORPHA:1496

Corpus callosum agenesis-neuronopathy syndrome is a neurodeg enerative disorder characterized by severe progressive sensorimotor neuropathy beginning in infancy with resulting hypotonia, areflexia, amyotrophy and variable degrees of dysgenesis of the corpus callosum. Additional features include mild-to-severe intellectual and developmental delays, and psychiatric manifestations that include paranoid delusions, depression, hallucinations, and "autistic-like" features. Affected individuals are usually wheelchair restricted in the second decade of life and die in the third decade of life. The disease is inherited as an autosomal recessive trait. 